\title{
T cell immunotherapy in hepatitis $B$ virus related hepatocellular carcinoma
}

\author{
Morteza Hafezi, Antonio Bertoletti, Anthony T. Tan \\ Emerging Infectious Diseases Programme, Duke-NUS Medical School, Singapore 169857, Singapore. \\ Correspondence to: Dr. Anthony T. Tan, Emerging Infectious Diseases Programme, Duke-NUS Medical School, 8 College Road, \\ Singapore 169857, Singapore. E-mail: anthony.tan@duke-nus.edu.sg \\ How to cite this article: Hafezi M, Bertoletti A, Tan AT. T cell immunotherapy in hepatitis B virus related hepatocellular carcinoma. \\ Hepatoma Res 2018;4:16. http://dx.doi.org/10.20517/2394-5079.2018.55
}

Received: 11 May 2018 First Decision: 22 May 2018 Revised: 22 May 2018 Accepted: 22 May 2018 Published: 30 May 2018

Science Editor: Guang-Wen Cao Copy Editor: Jun-Yao Li Production Editor: Huan-Liang Wu

\begin{abstract}
Chronic hepatitis B virus (HBV) infection is a major risk factor for hepatocellular carcinoma (HCC). While multiple treatment modalities are available, liver transplantation remains the sole curative treatment for advanced stages of HCC, and hence new treatment approaches are required to fulfill this unmet need of curative HCC therapy. Our first-in-man proof-of-concept adoptive T-cell immunotherapy against HBV related hepatocellular carcinoma metastases has shown promising results. Here, we review the development of T-cell immunotherapy targeting $\mathrm{HBV}$ antigens for the treatment of HBV-HCC and discuss the practical considerations for the safe and effective use in clinics.
\end{abstract}

Keywords: Chronic hepatitis B virus, hepatocellular carcinoma, T-cell immunotherapy

\section{INTRODUCTION}

Hepatocellular carcinoma (HCC) is the primary liver malignancy in adults, and it occurs predominantly in patients with chronic liver inflammation and cirrhosis. It accounts for approximately 800,000 deaths annually worldwide and in the majority of these cases, hepatocellular carcinoma (HCC) occurrence is linked to chronic hepatitis B virus (HBV) infection ${ }^{[1]}$. HBV is a non-cytopathic DNA virus from the Hepadnaviridae family that specifically infects hepatocytes. Patients with chronic HBV infection can remain largely asymptomatic, but viral persistence increases the risk of developing liver complications like fibrosis, cirrhosis and hepatocellular carcinoma ${ }^{[2,3]}$. Despite prophylactic vaccination against HBV, approximately 300 million people globally have been infected with this virus ${ }^{[2]}$ and among chronically infected individuals, approximately $25 \%$ will develop HCC neoplasm ${ }^{[4]}$.

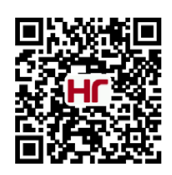


Due to the lack of effective diagnostic and screening strategies, most of the HCC patients are diagnosed late. Depending on the stage of the cancer, multiple different treatment modalities like transplantation, partial hepatectomy, chemo-embolization, systemic chemotherapy could be given ${ }^{[5,6]}$. Among these approaches, the only potentially available curative therapy is resection and liver transplantation which can only be applied in the early stages of HCC before metastasis are detected ${ }^{[5]}$. However, for the majority of patients who are diagnosed at later stages, or with metastases, current available therapy is ineffective and even first line drugs like sorafenib can only increase the survival for up to 3 months in these patients ${ }^{[7]}$. In addition to the lack of an effective therapy for the majority of HCC patients, the increasing supply of donor livers with the advent of living donor transplantation has resulted in a change of the liver transplantation criteria. New Criteria were developed to include patients with more advanced disease. Though this has opened up the option of liver transplantation for more HCC patients, it also has a negative impact on the post-transplantation HCC recurrence rates. In most cases, therapeutic options for patients who have tumour recurrence post liver transplantation are even more limited ${ }^{[8-11]}$. Therefore, there is a clear unmet need which supports the development of new effective therapeutic approaches. In this review, we focus on the use of adoptive T-cell immunotherapy targeting HBV antigens for the treatment of HBV-HCC and discuss the practical considerations for their use in clinics.

\section{T-CELL IMMUNOTHERAPY FOR HCC}

Immunotherapy has shown promising outcomes in different hematologic malignancies, demonstrating its high potential for curative HCC therapy ${ }^{[12,13]}$. Major progress have been made in the development of immunotherapy approaches that attempts to rejuvenate and/or induce anti-tumour $\mathrm{T}$ cell responses in the HCC microenvironment, like immune checkpoint inhibitors (ICIs) ${ }^{[14]}$. However, this approach requires a pre-existing inflammatory tumour microenvironment with significant immune cell infiltration, the expression of immune checkpoints on tumour cells, and/or an existing anti-tumour immune response, in order to exert an anti-tumour effect ${ }^{[13,15-18]}$. With the intra- and inter- HCC patient tumour heterogeneity, it would be difficult to expect the mechanism of action for the therapy to be intact for all tumour nodules, especially in metastatic nodules that develop in different anatomical environments ${ }^{[19]}$. Some tumours will be inherently devoid of infiltrating T-cells and hence will not respond to such treatments ${ }^{[20]}$.

Furthermore, this approach is non-specific. It aims to augment the general anti-tumour immune response. This comes with its own drawbacks as the enhanced immune response is a double edged sword. On one hand, it provides the desired anti-tumour effect, on the other, it could result in uncontrolled autoimmune effects $^{[21]}$. This is particularly important in patients with HCC recurrence post liver transplantation. In these patients, immunosuppressive agents are given to control graft rejection, but the very same enhanced anti-tumour response due to checkpoint inhibitors could also lead to uncontrolled inflammation and even graft rejection ${ }^{[22,23]}$, which is why the use of checkpoint inhibitors is at the moment not indicated for liver transplanted patients.

In such scenarios, the adoptive transfer of personalized autologous engineered $\mathrm{T}$ cells maybe a suitable strategy. Currently, multiple clinical trials using autologous engineered T cells against HCC are ongoing ${ }^{[24]}$. Unlike others, this strategy does not rely on the immune pre-requisites above, instead new anti-tumour T-cells are engineered in vitro and reinfused back into the patient to combat the tumour [Figure 1]. In addition, the extensive body of work involved in the development of CD19-specific T-cell immunotherapy for B-cell leukemia has clearly demonstrated the potent cytotoxic function of autologous engineered T-cell ${ }^{[25,26]}$. At present, adoptive T-cell therapy comprises of introducing either chimeric antigen receptors (CAR) or T-cell receptors (TCR) to re-direct the specificity of T cells towards the tumours, each with its own advantages or limitations.

CARs are membrane-bound proteins composed of an ectodomain, typically derived from a single-chain 


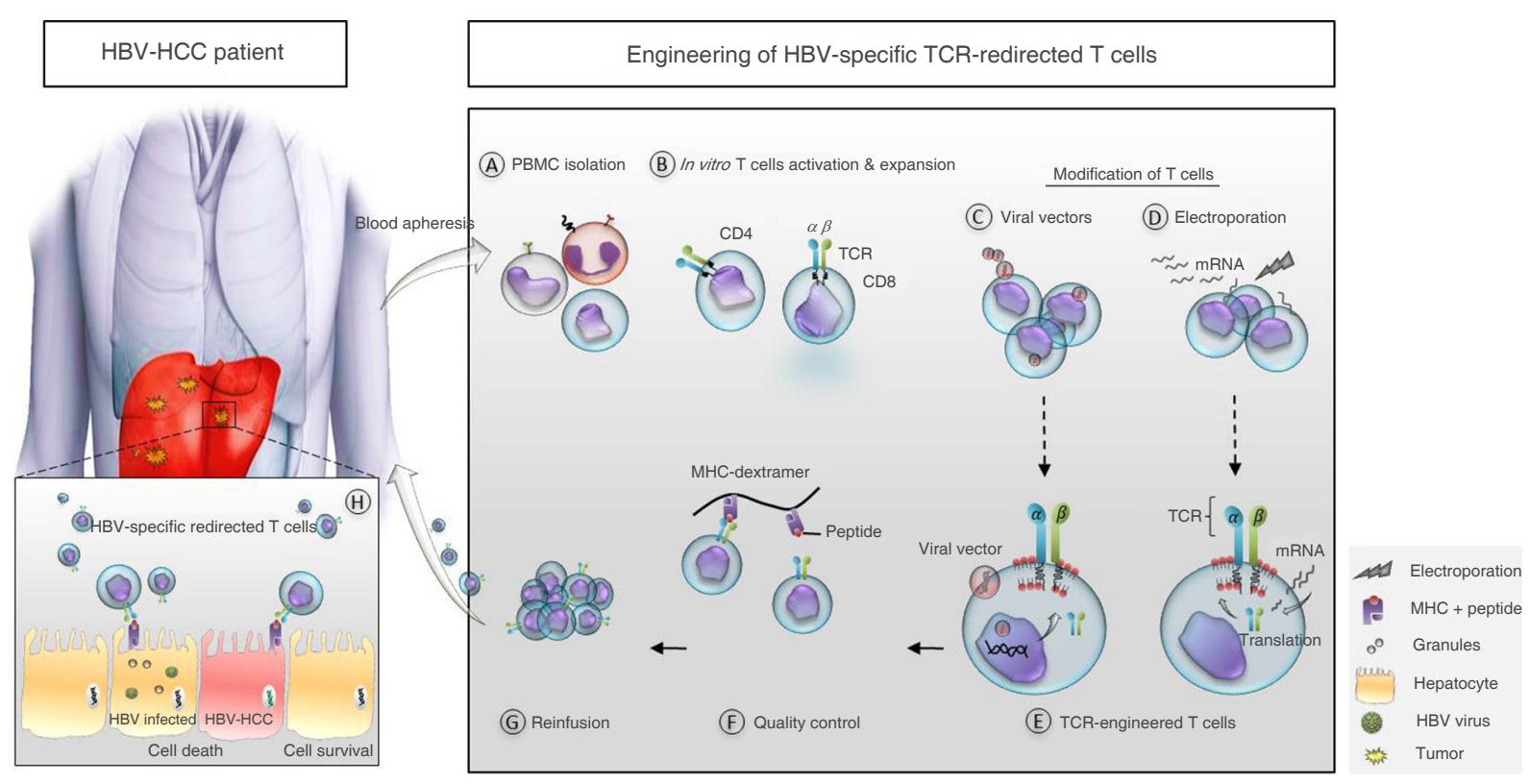

Figure 1. Schematic illustrating the production of personalized HBV-specific TCR redirected T cells. A: PBMC isolation from HCC patients; B: activation and expansion of $\alpha \beta$ TCR T cells for modification; C: transduce activated T cells with viral vectors encoding HBV-specific TCRs; D: electroporate activated T cells with in vitro transcribed mRNA; E: TCR-T cells engineered through viral transduction has the gene encoding the TCR integrated into the genome while electroporation only results in the translation of the introduced mRNA; F: analysis of the expression kinetics and function of HBV-specific TCR-T cells by tetramer staining and immune assays; G: adoptive transfer of autologous HBV-specific TCR-T cells back into the HBV-HCC patient; H: cytolysis of HBV expressing hepatocyte or HCC cells. HBV: hepatitis B virus; TCR: T-cell receptor; PBMC: peripheral blood mononuclear cell; HCC: hepatocellular carcinoma

variable fragment (ScFv), hinge and transmembrane domain. The ectodomain enables CARs to recognize cancer antigens in a HLA-independent manner. This particular feature enables CAR redirected T cells to be used in more patients without being restricted by their HLA haplotype, and also to target tumor cells that down-regulate their HLA expression ${ }^{[27,28]}$. However, CAR recognition is limited only to conformationally intact antigens, both cell membrane bound or soluble forms. This represents only a small fraction of the total cellular proteins which limits the pool of antigens that can be targeted by CARs. In contrast, the TCR consist of alpha-beta chain heterodimeric glycoprotein which recognizes almost any degraded intracellular protein via the HLA system. This means that a greater degree of personalization is required when applied in patients, but at the same time, a larger number of tumour-specific $\mathrm{T}$ cell epitopes could potentially be targeted ${ }^{[29]}$.

These advantages of T-cell immunotherapy makes it a highly promising approach as a curative HBVHCC treatment. However, choosing the appropriate tumour-specific antigen to redirect the T-cells towards remains a critical decision that dictates both the efficacy and safety of the approach.

\section{TARGETING HBV ANTIGENS AS A TUMOUR ANTIGEN}

Several clinical trials have shown that both CAR and TCR redirected T-cell therapy can cause substantial solid tumour regression ${ }^{[30]}$. In all these cases, tumour discrimination is determined by the recognition of classical tumour associated antigens (TAA; alpha-fetoprotein, NY-ESO, MAGE, EGFR), essentially selfantigens that are aberrantly expressed in tumour cells, by high affinity CARs or TCRs. Such aberrant expression includes the overexpression of certain cell surface proteins at high levels, or the expression of fetal antigens that are typically not found in normal cells at a steady state ${ }^{[31]}$. In both cases, due to the selfnature of the TAA, one cannot reliably predict and hence exclude the expression of the supposed tumourspecific antigens on other healthy cells. Adult cells undergoing active division could re-express fetal antigens which otherwise remains non-expressed when at a steady state. This is made even more challenging when high affinity CARs or TCRs, which recognizes pico-molar quantities of TAA, are used. For instance, clinical trials from NCI and Adaptimmune has shown the unexpected binding of high affinity MAGE-A3 TCR to similar epitopes like MAGE-A12 and titin in the brain and heart respectively, resulting in severe off-tumour 
responses and a fatal outcome ${ }^{[29]}$. Targeting overexpressed self-antigens in tumours might not be ideal due to potential off-tumour responses against normal cells ${ }^{[29,32]}$.

Recently, the discovery of altered self-antigens in highly mutated tumours have sparked an interest in their use as a TAA for T-cell immunotherapy ${ }^{[33]}$. The continuous evolution and accumulation of mutations in tumours can result in the modification of self-antigens to an extent where they are no longer perceived as self-antigens, and hence become immunogenic. These neoantigens have been reported to be capable of inducing a robust T-cell response which mediates an anti-tumour effect. Since neoantigens are sufficiently different from self-antigens, this makes them a better TAA with far lower risks of on-target off-tumour responses observed when targeting classical TAAs. However, the generation of neoantigens involves the accumulation of random mutations which differs between tumour nodules and patients, making their discovery and characterization difficult and cumbersome ${ }^{[34]}$.

A possible alternative approach is to target HBV antigens as a TAA. In the natural history of chronic HBV infection, the virus integrates itself into the human genome, hence the HCC cells that eventually develop from chronically infected hepatocytes will carry these integrations and can be targeted by HBV-specific TCR

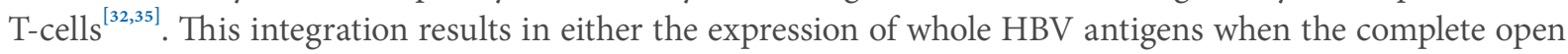
reading frame is integrated, or the production of chimeric HBV-host proteins when only short fragments of HBV are integrated ${ }^{[36]}$. In any case, the integration process inadvertently marks the HBV-HCC cells with a foreign antigen through a mechanism that is highly hepatotropic as dictated by the infectivity of $\mathrm{HBV}^{[32]}$. This liver-specific marking would mean that the on-target off-tumour adverse events is largely predictable and would primarily be limited to the liver compartment, with little or no involvement of other organs ${ }^{[29]}$. However, since HBV-specific TCR-T cells are unable to discriminate between HBV-infected hepatocytes and HBV-HCC cells, the risk of on-target off-tumour lysis of infected hepatocytes is also of concern. At present, this issue can be circumvented by treating only HBV-HCC patients with tumour recurrence post liver transplantation and by selecting HBV-specific TCRs restricted by HLA molecules present on the patient cells and not on the donor liver. Extending this approach to HBV-HCC patients without liver transplantation will have to be properly evaluated after more clinical data and experience have been accumulated. In addition to the desirable safety considerations above, targeting HBV antigens would also provide a commonality across multiple tumour nodules and patients, unlike the diverse and somewhat random nature of neoantigens, making it less complicated to use in clinics.

It is also important to note that the targeting of HBV antigens for HBV-HCC treatment comes with its own restrictions due to the natural virological characteristics of chronic HBV infection. In chronic HBV patients, microgram quantities of HBV envelope antigens are circulating in the serum. These soluble HBV antigens can interfere with the function of HBV-envelope specific CAR-T cells by either blocking and sequestering of the cell surface CARs, or by the inappropriate activation of the CAR-T cells ${ }^{[32,37,38]}$. However, the obligate requirement for HLA presentation of T cell epitopes to TCRs would render the TCR-T cells insensitive to soluble HBV antigens in the serum ${ }^{[39]}$. To capitalise on the better safety considerations associated with targeting HBV antigens, one would have to employ the use of HBV-specific TCRs and not CARs recognizing HBV antigens for HBV-HCC T-cell immunotherapy.

\section{FIRST-IN-MAN PROOF-OF-CONCEPT TCR-T CELL IMMUNOTHERAPY OF HBV-HCC}

The feasibility of using HBV-specific TCR-T cells for the treatment of HBV-HCC was first demonstrated in a compassionate therapy of a chronic HBV patient who has widespread extrahepatic HCC metastasis post-liver transplantation ${ }^{[40]}$. The combination of several clinical features of the patient makes him an ideal candidate for the first-in-man proof-of-concept therapy where an emphasis on safety is essential. First, the patient had undergone liver transplantation. This means that the main bulk of HBV infected 
hepatocytes have been removed, reducing the risk of overt destruction of functional hepatocytes. This risk is further lowered by confirming the absence of HBcAg, HBsAg and HBV DNA from a biopsy sample of his transplanted liver. Second, immunohistochemistry analysis of his metastatic tumour nodules shows the expression of HBsAg. This not only suggest that the tumour cells can be recognized by HBV-specific TCR-T cells, it also means that the serum levels of HBsAg could be used as a surrogate to monitor the efficacy of therapy as HBsAg is only produced by the tumour cells. With a single infusion of small numbers $\left(10^{4} \mathrm{HBV}\right.$ specific TCR-T cells $/ \mathrm{kg}$ ) of retroviral transduced TCR-T cells, the cells expanded efficiently in vivo ( $2 \%$ of CD8 T cells), and a reduction of over $90 \%$ of the serum HBsAg levels was achieved within 30 days without exacerbation of liver inflammation or any detectable on/off-target toxicities. This was not observed over the duration of one year when multiple radiotherapy and surgical resections of the tumours were performed. Unfortunately, the patient was treated at a very late stage and he succumbed to his disease after 8 weeks of monitoring. Nonetheless, the promising results obtained from this proof-of-concept therapy warranted further development of this treatment approach.

\section{PRACTICAL CONSIDERATIONS FOR SAFE AND EFFECTIVE T CELL IMMUNOTHERAPY OF HBV-HCC}

Despite the encouraging data obtained from the proof-of-concept therapy described above, additional considerations will have to be addressed in order to develop a safe and effective TCR-T cell immunotherapy for HBV-HCC. The first consideration is the issue of safety associated with the use of viral vectors for the delivery of the TCR gene construct. The oncogenic effect mediated by the insertion of the TCR gene into the host genome is a potential concern. More importantly, viral transduction generates $\mathrm{T}$ cells that stably expresses the HBV-specific TCR, allowing them to expand in vivo. This in vivo persistence may be beneficial for tumour eradication, but it would also pose a safety concern as the quantity and function of the modified $\mathrm{T}$ cells could not be easily controlled if a treatment related adverse event were to occur ${ }^{[32]}$. An alternative is to introduce the TCR gene via the electroporation of in vitro transcribed functional mRNA [Figure 1] ${ }^{[41]}$. This approach will not result in insertional mutagenesis and the expression of the introduced TCR is transient, while maintaining the anti-tumour effects. Not only will you have better control of the TCR-T cell function, the transient expression also allows clinical trials to be designed with an intra-patient dose-escalation protocol and thereby improving the safety. At the moment, HBV-specific TCR-T cells modified through mRNA electroporation have been extensively characterized in vitro and in in vivo pre-clinical models and is currently utilized in clinical trials for the treatment of HBV-HCC in liver transplanted patients ${ }^{[42]}$. In addition, the transient function of mRNA electroporated $\mathrm{T}$ cells is ideal for the treatment of the majority of HBV-HCC patients who have not undergone liver transplantation, where the risk of on-target off-tumour lysis of functional but HBV infected hepatocytes is high.

Patient selection is also a critical issue that needs to be addressed. Barring inclusion and exclusion criteria associated with clinical parameters, at the moment, patient eligibility is dictated solely by the HLA haplotype of the patient ${ }^{[29]}$. In which case, the patient is suitable for therapy if he/she expresses the appropriate HLA molecule capable of presenting the T-cell epitope recognized by the TCR-T cells. This simplistic criteria only takes into consideration the HLA component of the complex recognized by the TCRs ${ }^{[29]}$ For the therapy to be effective, one has to be able to account for the presence or absence of the T cell epitope on the HCC cells. Ideally, this can be achieved using TCR-like antibodies specific for every HLA/HBV-epitope complex ${ }^{[43,44]}$ but the diversity of complexes makes this approach unfeasible ${ }^{[45]}$. Peptide elution and mass spectrometry strategies ${ }^{[46]}$ might seem possible, but such techniques is highly specialized and complex, and at the moment restricted primarily to academic research and not clinical application. As a compromise, the detection of HBV proteins, DNA or mRNA would suffice with the assumption that antigen processing and epitope presentation occurs as expected. This is simpler in the situation where the complete open reading frame of a HBV antigen is integrated in the HCC cells. Detection of HBV antigens by serological means through immunohistochemistry analysis of tumour tissues would be sufficient. However, a recent study demonstrated 
the existence of HBV-host chimeric proteins in $\mathrm{HCC}^{[47-49]}$, where only short fragments of HBV DNA are integrated into the host genome. In such situations, serological assays will fail to detect the presence of HBV antigens and the patient would be deemed unsuitable for TCR-T immunotherapy. However, these chimeric proteins could potentially be processed and presented on the HCC cell surface, rendering the tumours recognizable by TCR-T cells. These integrations could only be detected through genetic means. As such, to have better patient selection, it is essential to develop new genetic based assays for the rapid detection of short HBV integrations and determine whether the appropriate HBV T-cell epitopes could be potentially produced by HBV-HCC cells.

Lastly, it is also necessary to understand how the basal biochemical parameters of HBV-HCC patients could influence the function of TCR-T cells. It is common to have HBV-HCC patients with, elevated serum alphafetoprotein levels, to be treated with multi-kinase inhibitors or with immunosuppressive agents if they have been liver transplanted. The effects of such variables have remained largely unexplored in the context of TCR-T cell immunotherapy, but it could have important impacts on the treatment efficacy.

\section{CONCLUSION}

In this short review, we have discussed the need for new treatment strategies against HBV-HCC, the scientific rationale that guides the development of HBV-specific TCR-T cell immunotherapy and some practical considerations surrounding its use in patients. It is in our opinion that many unknowns still remain. At the moment, dosing and infusion frequencies are still determined arbitrarily, or extrapolated from T-cell immunotherapies for other cancers, while the accessibility of tumours at different anatomical locations, and even the function of TCR-T cells in different tumour microenvironments remains a subject of continuous investigation. We are however confident that the promising potential of T-cell immunotherapy will stimulate further research and development making its use in the treatment of HBV-HCC a reality.

\section{DECLARATIONS}

\section{Authors' contributions}

Hafezi M, Bertoletti A and Tan AT wrote the manuscript.

\section{Financial support and sponsorship}

This work was supported by a Singapore Translational Research (STaR) Investigator Award (NMRC/ STaR/013/2012) to Bertoletti A.

\section{Conflicts of interest}

Bertoletti A participates in Advisory Boards on hepatitis B virus immune therapy for Gilead, Janssen, Medimmune and is a co-founder of Lion TCR Private Limited, a biotech company developing T cell receptors for treatment of virus-related cancers and chronic viral diseases. Tan AT is a consultant of Lion TCR Private Limited. Hafezi M discloses no conflicts.

\section{Patient consent}

Not applicable.

\section{Ethics approval}

Not applicable.

\section{Copyright}

(c) The Author(s) 2018. 


\section{REFERENCES}

1. Ringelhan M, Pfister D, O’Connor T, Pikarsky E, Heikenwalder M. The immunology of hepatocellular carcinoma. Nat Immunol 2018;19:222-32.

2. Liang TJ. Hepatitis B: the virus and disease. Hepatology 2009;49:S13-21.

3. Liaw YF, Chu CM. Hepatitis B virus infection. Lancet 2009;373:582-92.

4. Ganem D, Prince AM. Hepatitis B virus infection--natural history and clinical consequences. N Engl J Med 2004;350:1118-29.

5. Raza A, Sood GK. Hepatocellular carcinoma review: current treatment, and evidence-based medicine. World J Gastroenterol 2014;20:411527.

6. Mlynarsky L, Menachem Y, Shibolet O. Treatment of hepatocellular carcinoma: steps forward but still a long way to go. World J Hepatol 2015;7:566-74.

7. Llovet JM, Ricci S, Mazzaferro V, Hilgard P, Gane E, Blanc JF, de Oliveira AC, Santoro A, Raoul JL, Forner A, Schwartz M, Porta C, Zeuzem S, Bolondi L, Greten TF, Galle PR, Seitz JF, Borbath I, Haussinger D, Giannaris T, Shan M, Moscovici M, Voliotis D, Bruix J; SHARP Investigators Study Group. Sorafenib in advanced hepatocellular carcinoma. N Engl J Med 2008;359:378-90.

8. Lee SG. Living-donor liver transplantation in adults. Br Med Bull 2010;94:33-48.

9. Saab S, Yeganeh M, Nguyen K, Durazo F, Han S, Yersiz H, Farmer DG, Goldstein LI, Tong MJ, Busuttil RW. Recurrence of hepatocellular carcinoma and hepatitis B reinfection in hepatitis B surface antigen-positive patients after liver transplantation. Liver Transpl 2009;15:152534.

10. Lee JO, Kim DY, Lim JH, Seo MD, Yi HG, Oh DY, Im SA, Kim TY, Bang YJ. Palliative chemotherapy for patients with recurrent hepatocellular carcinoma after liver transplantation. J Gastroenterol Hepatol 2009;24:800-5.

11. Rahimi RS, Trotter JF. Liver transplantation for hepatocellular carcinoma: outcomes and treatment options for recurrence. Ann Gastroenterol 2015;28:323-30.

12. Pardee AD, Butterfield LH. Immunotherapy of hepatocellular carcinoma: unique challenges and clinical opportunities. Oncoimmunology 2012;1:48-55.

13. Breous E, Thimme R. Potential of immunotherapy for hepatocellular carcinoma. J Hepatol 2011;54:830-4.

14. Bertoletti A, Bert NL. Immunotherapy for chronic hepatitis B virus infection. Gut Liver 2018; doi: 10.5009/gnl17233.

15. Wan S, Kuo N, Kryczek I, Zou W, Welling TH. Myeloid cells in hepatocellular carcinoma. Hepatology 2015;62:1304-12.

16. Kahan SM, Wherry EJ, Zajac AJ. T cell exhaustion during persistent viral infections. Virology 2015;479-480:180-93.

17. Pfirschke C, Engblom C, Rickelt S, Cortez-Retamozo V, Garris C, Pucci F, Yamazaki T, Poirier-Colame V, Newton A, Redouane Y, Lin YJ, Wojtkiewicz G, Iwamoto Y, Mino-Kenudson M, Huynh TG, Hynes RO, Freeman GJ, Kroemer G, Zitvogel L, Weissleder R, Pittet MJ. Immunogenic chemotherapy sensitizes tumors to checkpoint blockade therapy. Immunity 2016;44:343-54.

18. Vonderheide RH. The immune revolution: a case for priming, not checkpoint. Cancer Cell 2018;33:563-9.

19. Villanueva A, Llovet JM. Impact of intra-individual molecular heterogeneity in personalized treatment of hepatocellular carcinoma. Hepatology 2012;56:2416-9.

20. Teng MW, Ngiow SF, Ribas A, Smyth MJ. Classifying cancers based on T-cell infiltration and PD-L1. Cancer Res 2015;75:2139-45.

21. Byun DJ, Wolchok JD, Rosenberg LM, Girotra M. Cancer immunotherapy - immune checkpoint blockade and associated endocrinopathies. Nat Rev Endocrinol 2017;13:195-207.

22. Johnson DB, Sullivan RJ, Menzies AM. Immune checkpoint inhibitors in challenging populations. Cancer 2017;123:1904-11.

23. Spain L, Higgins R, Gopalakrishnan K, Turajlic S, Gore M, Larkin J. Acute renal allograft rejection after immune checkpoint inhibitor therapy for metastatic melanoma. Ann Oncol 2016;27:1135-7.

24. Johnson LA, June CH. Driving gene-engineered T cell immunotherapy of cancer. Cell Res 2017;27:38-58.

25. Sadelain M, Riviere I, Riddell S. Therapeutic T cell engineering. Nature 2017;545:423-31.

26. D'Aloia MM, Zizzari IG, Sacchetti B, Pierelli L, Alimandi M. CAR-T cells: the long and winding road to solid tumors. Cell Death Dis 2018;9:282.

27. Chmielewski M, Hombach AA, Abken H. Antigen-specific T-cell activation independently of the MHC: chimeric antigen receptorredirected T cells. Front Immunol 2013;4:371.

28. Li K, Lan Y, Wang J, Liu L. Chimeric antigen receptor-engineered T cells for liver cancers, progress and obstacles. Tumour Biol 2017;39:1010428317692229.

29. Garber K. Driving T-cell immunotherapy to solid tumors. Nat Biotechnol 2018;36:215-9.

30. Barrett DM, Grupp SA, June CH. Chimeric antigen receptor- and TCR-modified T cells enter main street and wall street. J Immunol 2015;195:755-61.

31. Hinrichs CS, Restifo NP. Reassessing target antigens for adoptive T-cell therapy. Nat Biotechnol 2013;31:999-1008.

32. Bertoletti A, Brunetto M, Maini MK, Bonino F, Qasim W, Stauss H. T cell receptor-therapy in HBV-related hepatocellularcar cinoma. Oncoimmunology 2015;4:e1008354.

33. Schumacher TN, Schreiber RD. Neoantigens in cancer immunotherapy. Science 2015;348:69-74.

34. The problem with neoantigen prediction. Nat Biotechnol 2017;35:97.

35. Bertoletti A, Tan AT, Koh S. T-cell therapy for chronic viral hepatitis. Cytotherapy 2017;19:1317-24.

36. Tu T, Budzinska MA, Shackel NA, Urban S. HBV DNA integration: molecular mechanisms and clinical implications. Viruses 2017;9:E75.

37. Eshhar Z. Tumor-specific T-bodies: towards clinical application. Cancer Immunol Immunother 1997;45:131-6.

38. Kruse RL, Shum T, Tashiro H, Barzi M, Yi Z, Whitten-Bauer C, Legras X, Bissig-Choisat B, Garaigorta U, Gottschalk S, Bissig KD. HBsAg-redirected T cells exhibit antiviral activity in HBV-infected human liver chimeric mice. Cytotherapy 2018;20:697-705.

39. Gehring AJ, Xue SA, Ho ZZ, Teoh D, Ruedl C, Chia A, Koh S, Lim SG, Maini MK, Stauss H, Bertoletti A. Engineering virus-specific T cells that target HBV infected hepatocytes and hepatocellular carcinoma cell lines. J Hepatol 2011;55:103-10. 
40. Qasim W, Brunetto M, Gehring AJ, Xue SA, Schurich A, Khakpoor A, Zhan H, Ciccorossi P, Gilmour K, Cavallone D, Moriconi F, Farzhenah F, Mazzoni A, Chan L, Morris E, Thrasher A, Maini MK, Bonino F, Stauss H, Bertoletti A. Immunotherapy of HCC metastases with autologous T cell receptor redirected T cells, targeting HBsAg in a liver transplant patient. J Hepatol 2015;62:486-91.

41. Koh S, Shimasaki N, Suwanarusk R, Ho ZZ, Chia A, Banu N, Howland SW, Ong AS, Gehring AJ, Stauss H, Renia L, Sallberg M, Campana D, Bertoletti A. A practical approach to immunotherapy of hepatocellular carcinoma using T cells redirected against hepatitis B virus. Mol Ther Nucleic Acids 2013;2:e114.

42. TCR-Redirected T Cells Infusions to Treat Patients With Recurrent Hepatocellular Carcinoma Post Liver Transplantation. In: ClinicalTrials. gov, Identifier: NCT02719782.

43. Dahan R, Reiter Y. T-cell-receptor-like antibodies - generation, function and applications. Expert Rev Mol Med 2012;14:e6.

44. Sastry KS, Too CT, Kaur K, Gehring AJ, Low L, Javiad A, Pollicino T, Li L, Kennedy PT, Lopatin U, Macary PA, Bertoletti A. Targeting hepatitis B virus-infected cells with a T-cell receptor-like antibody. $J$ Virol 2011;85:1935-42.

45. Chang AY, Gejman RS, Brea EJ, Oh CY, Mathias MD, Pankov D, Casey E, Dao T, Scheinberg DA. Opportunities and challenges for TCR mimic antibodies in cancer therapy. Expert Opin Biol Ther 2016;16:979-87.

46. Bassani-Sternberg M, Pletscher-Frankild S, Jensen LJ, Mann M. Mass spectrometry of human leukocyte antigen class I peptidomes reveals strong effects of protein abundance and turnover on antigen presentation. Mol Cell Proteomics 2015;14:658-73.

47. Chiu YT, Wong JK, Choi SW, Sze KM, Ho DW, Chan LK, Lee JM, Man K, Cherny S, Yang WL, Wong CM, Sham PC, Ng IO. Novel premRNA splicing of intronically integrated HBV generates oncogenic chimera in hepatocellular carcinoma. J Hepatol 2016;64:1256-64.

48. Hino O, Kitagawa T, Koike K, Kobayashi M, Hara M, Mori W, Nakashima T, Hattori N, Sugano H. Detection of hepatitis B virus DNA in hepatocellular carcinomas in Japan. Hepatology 1984;4:90-5.

49. Tokino T, Matsubara K. Chromosomal sites for hepatitis B virus integration in human hepatocellular carcinoma. J Virol 1991;65:6761-4. 